\title{
Research on Unified Application Development Framework for Real Time Data Center
}

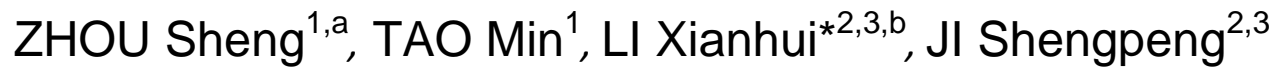 \\ ${ }^{1}$ Electric Power Research Institute, State Grid Zhejiang Electric Power Company, Hangzhou 310014 \\ NARI Group Corporation, Nanjing, 210000,China) \\ ${ }^{3}$ China Realtime Database Co.,Ltd.,Nanjing, 210000, China \\ a zhousheng851114@163.com, ${ }^{\text {b }}$ lixianhui@sgepri.sgcc.com.cn,
}

\begin{abstract}
Real Time Data Center is a centralized place for time sequence data storage and management. With the continuous improvement of the practical level of Real Time Data Center , more and more applications are developed based on it. However, the applications upper on the Real Time Data Center are lack of a unified technical architecture, resulting in the development of time-consuming and difficult management. In view of the above situation, this paper presents a kind of real-time data center unified application development framework. Standardized application development from technology and management. It provides a new idea for future application development, operation and maintenance management based on the Real Time Data Center.
\end{abstract}

Keywords: Real Time Data Center. Application development framework. Technical framework. Specification.

\section{Introduction}

With the development of electric power enterprises strong smart grid construction step by step, produced a large number of real-time data, and then precipitate the massive historical data, massive real-time / historical data are important wealth in the process of production and operation of power grid enterprises, and also an important information foundation of lean management. In this context, the Zhejiang Electric Power Corporation actively explore and try to establish the real-time / historical data platform based on domestic real time database as the foundation, in order to meet the needs of the business application of real-time / historical data storage, integration, sharing and unified and standard access. However, there is still a lack of unified planning platform at the application level, it is necessary to form a unified application technology framework.

\section{Situation analysis}

The real-time / historical data platform is formed on the power grid operation process in the application of the real-time / historical data storage, centralized, integration, sharing and analysis of the tool, also provides a standard unified access mode, for the smart grid and the each application of SG-ERP. Especially interdisciplinary, cross sectoral integrated business application in the information infrastructure of real-time / historical data level to provide technical support[1-2] .

At present, the domestic and foreign technical framework for real-time / historical data application mainly focuses on data management, such as data access, storage, access and other aspects of the technical framework and technical standards. There are no relevant specifications and standards for the application technology framework.

Therefore, from centralized, unified and standardized management platform application requirements, considering the sustainable development platform,in the "unified application platform" standardized management level to deal with platform application framework.

\section{Unified application architecture}

\subsection{Overall architecture}

Zhejiang power grid real-time / historical data platform using the province's centralized deployment mode. In order to promote the standardized development platform application, especially the unified planning and implementation of the provincial comprehensive application, should adopt the unified architecture application technology, application technology framework in application platform construction on the development unit must follow the unified platform standard application, 
makes the business of different functional modules will be packaged into a run in the environment. The overall architecture of the application platform is shown in figure 1.

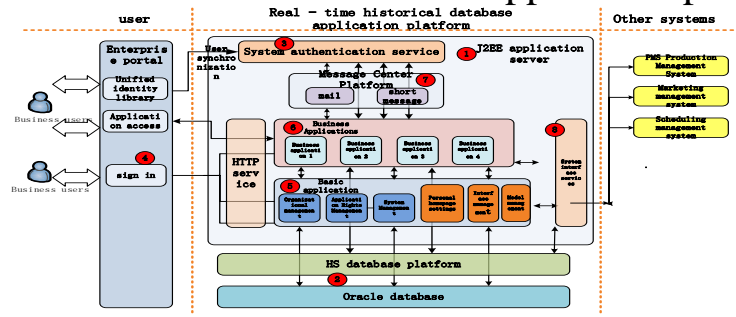

Fig.1 The architecture of application platform

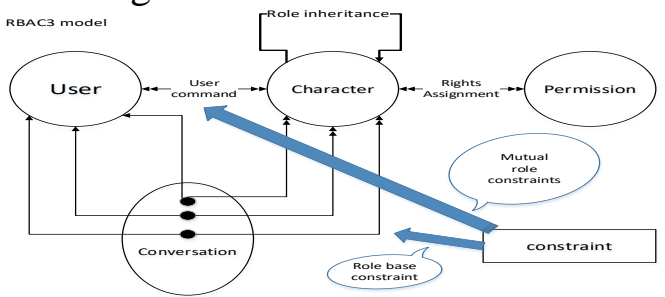

Fig.2 RBAC3 model

The architecture has the following features.

(1) J2EE architecture. Application platform based on J2EE architecture, all application modules will run in a unified Web APP.

(2) Database. Application of real-time database and relational database based, real-time / historical data are stored in real-time database, the application through the real-time database provided by API, SDK to achieve access to real-time / historical data. In the application platform, the relational database is used to store and manage the user, the organization structure, the application permission and the metadata, which are closely related to the specific business.

(3) System authentication service. Business users access to the application system must provide authentication services through the platform, only legitimate users can access the corresponding business module.

(4) Single sign on. The system realizes the integration with the enterprise portal system, and realizes the user single sign on, after the user enters the enterprise portal, does not need to enter the user name and the password, may visit the integrated application system directly.

(5) Basic business applications. The application of the system platform includes the management of the organization, the application of authority management, system management, personal home page settings and point of management and other functional modules. Personnel management: mainly used to provide coverage of the province's business units of the organizational structure, as well as the corresponding user information. Application rights management: the role of the system to provide users with the distribution function, and all business modules related to the function of the configuration and binding authority. System management: provide service monitoring, logging, activity audit and system parameter configuration for the specific business application system. Personal home page settings: mainly to provide users with personalized home settings, including some user personalization parameters. The measuring point: management is the extension of the real-time / historical data platform for the original point management function, maintain the basic information of measurement points in a relational database, provide the corresponding relationship between equipment real-time database of measurement points and transport inspection, marketing and regulation of professional platform, while providing maintenance point relationships, and can bind permission.

(6) Business applications by category. The future application of information management platform will be widely used in transport inspection, control, marketing, planning and auxiliary information resources monitoring business, these business function modules will be based on the real-time / historical data platform, a unified infrastructure, including organization, personnel, management authority, measuring points, for the professional custom function.

(7) System interface services. Application platform will provide a unified system interface services, external business systems (such as transportation, marketing, control systems) to provide real-time / historical data and related business functions. Monitoring and auditing of interface services.

\subsection{Application rights management}

In the real-time / historical data application platform, the role based access control (Role-Based Access Control, RBAC) is used to control the application rights. In the RBAC, including user, role, permission and other basic data elements, permission was given to the role, rather than the user, when 
a character is assigned to a user, the user will have access to [6-8] the role included. Session Sessions is a mapping between a user and an active set of roles. The RBAC3 model is shown in figure 2 .

In the traditional RBAC model, RBAC3 model introduces 2 kinds of characteristics: role inheritance and responsibility separation. The permission control of RBAC3 model has the following characteristics.

(1) The authority assignment relationship can be dynamically adjusted and modified according to the demand and the temporary situation. Because permissions are associated with roles, users get permission to get these roles by becoming members of the appropriate role, which greatly simplifies the management of permissions. In an organization, the role can be established based on the work of the business, the user will be based on the corresponding responsibilities and qualifications to assign the role, the user can easily be assigned from one role to another role. The role can be granted new permissions according to the new requirements and the merger of the system, and permissions can also be recovered from a role as needed.

(2) Role inheritance. In addition to its own attributes and permissions, but also can inherit other roles, which automatically has inherited the role of the properties and permissions. Such as paying users can inherit the attributes of ordinary users and permissions, super administrator can inherit the attributes and permissions of the general manager.

(3) Role constraint. The constraint principle will be enforced when the authority is assigned a role, or when the role is given to the user, and when the user activates a role at a certain time. The principle of constraint includes the role of mutual exclusion, that is to say, mutually exclusive roles cannot be active at the same time.

(4) Provides control over the instance level object. In RBAC model, resource access control object is the goal, the resources and the role assignment after binding will determine the user to access [9-10] resources, so the particle definition and classification resources in the application in the platform determines the fine degree of access control system.

\subsection{Point management}

The application of the measurement point management in the application platform is to establish the corresponding relationship between the real-time database measurement points and other external business system equipment models, and establish the related equipment object metadata. The corresponding model of the device is shown in figure 3.

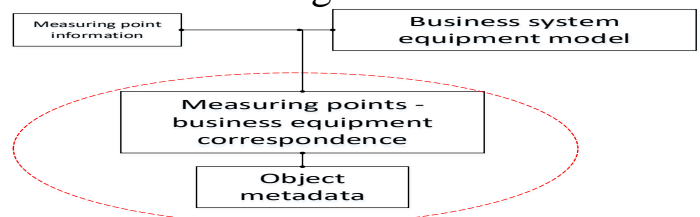

Fig.3 The mapping between equipments and models

The external business system is mainly production system, marketing system and scheduling system, equipment management model, these systems have their own independent, when real-time / historical data interface server platform access device data, if only to establish the corresponding relationship between the equipment and the measuring points, the corresponding end can not form the equipment model between different business systems the metadata (including equipment, equipment type, voltage level, subordinate units, substation, interval, equipment maintenance management, name, etc.) can be measured to determine the important metadata consistent with the data acquired from the resources of the same equipment, for the future of cross business system offers the basic business function design. At the same time, through the establishment of object metadata, it can be more convenient for the measurement of objects according to the metadata (such as voltage level, equipment type, etc.).

The main functions of this part are as follows.

(1) The corresponding relationship between the measurement points is established automatically. When the system collects data from the interface, according to the principle of the pre established equipment matching, it can automatically create the corresponding relationship between the measuring point and the equipment to meet the requirements of the equipment, and extract the 
equipment metadata information. Equipment metadata information includes equipment type, voltage level, substation, subordinate unit, data belongs to the business system, equipment name, etc..

(2) Provide the manual way to maintain the information of the measuring point and the metadata of the equipment, including adding, deleting, modifying and querying information.

(3) To provide data for the classification of statistical statements, for the analysis of the construction of measurement points.

(4) To provide the measurement of the corresponding relationship and import and export metadata, data formats can be used CSV or XML.

\subsection{System interface services}

The real-time / historical data platform to provide a range of data access service for the external system, the data related to the range of data, including power consumption data, equipment monitoring data, operation data, electric power quality data, meteorological data, lightning monitoring data, power operation data and other historical / quasi real time data.

Application platform to provide Web Service services outside the system, using a standard cross platform approach to provide data access services [11-12], specific functions are as follows.

(1) The Web Service service provides support for user authentication, the user needs to meet the data access permissions in the application platform, the user can not access unauthorized data.

(2) Provide a variety of historical / real-time data query services, including the latest data query, historical data query, historical data statistics, alarm time query, etc..

(3) Can be set to enable or disable the specified data access service.

(4) Enable or disable the service visitors.

(5) We can analyze the data access, analyze and control the service which may cause the performance problem.

\section{4. conclusion}

Through the analysis of the real-time/historical data platform application status, meet the uniform technical framework of each business application requirements, one can enhance the real-time/historical data application platform overall control ability, change the status quo and existing business application platform of the lack of unified planning and technical architecture specification, change the existing application technology to support the status quo manufacturers lack of coordination and there are technical barriers, forming a benign ecological chain platform construction. on the other hand can enhance the level of information real-time/historical data application platform, effective precipitation and accumulation of technology and service capabilities, enhance the level of practical application, is conducive to the sustainable development ability of application platform.

\section{5. references}

[1] FANG Ming-xia. Application situation and prospect of PI in Zhejiang power grid[J]. Zhejiang Electric Power, 2010, 29(4): 51-54.

[2] WANG Qiao-wen, TAO Wen-wei, DING Jian-yong, et al. Design and development of power supply enterprise real-time data center system based on PI database[J]. Automation of Electric Power Systems, 2009, 33(6): 99-103.

[3] FAN Yong, XU Xiao-zhong, YAN Hao-jun, et al. Real-time data management methods and tools for power grid enterprises[J]. East China Electric Power, 2012, 40(3): 485-487.

[4] Zhang Ying, Tang Lei. Design of data interface and communication protocol between SCADA and PI[C]. Proceedings of the 2006 Symposium on academic exchanges in automation of electric power systems. 2006: 195-197.

[5] TIAN Jia-ying, ZHAO Fang. Application of PI real-time database in power supply enterprise[J]. Power System Protection and Control, 2006, 34(15): 46-49.

[6] ZHANG Yan-yan. Implement of role-based access control[J]. Network Security Technology and Application, 2006(8): 53-54.

[7] IANG Yu- $\mathrm{f}$ eng, FU Yu, WU Xi ao-ping. De s ign and implementation of authorization system based on RBAC[J]. Computer and Digital Engineering, 2009, 37(6): 98-101. 
[8] DONG Yong-feng, LU Jun, LIU Jian-bo, et al. Application of improved RBAC model to information service platform[J]. Computer Applications and Software, 2012, 29(5): 99-103.

[9] WU Jiang-dong, LI Wei-hua, AN Xi-feng. Method of finely granular access control based on RBAC[J]. Computer Engineering, 2008, 34(20): 52-54.

[10] ZHAO Wei-dong, BI Xiao-qing, LU Xin-ming. Design and implementation of fine-grained RBAC model[J]. Computer Engineering and Design, 2013, 34(2): 474-479.

[11] TAO Min, GUO Ning. Optimization of platform architecture for plant information database[J]. Zhejiang Electric Power, 2011, 30(8): 1-4.

[12] ZHOU Sheng, TAO Min. Study of universal access method in real-time/historical database platform[J]. Zhejiang Electric Power, 2012, 31(12): 94-98. 